\title{
PRECISE ASYMPTOTICS IN COMPLETE MOMENT CONVERGENCE FOR DEPENDENT RANDOM VARIABLE
}

\author{
KWANG-HeE HAN
}

\begin{abstract}
Let $X, X_{1}, X_{2}, \cdots$ be identically distributed and negatively associated random variables with mean zeros and positive, finite variances. We prove that, if $E\left|X_{1}\right|^{r}<\infty$, for $1<p<2$ and $r>1+\frac{p}{2}$, and $\lim _{n \rightarrow \infty} n^{-1} E S_{n}^{2}=\sigma^{2}<\infty$, then

$$
\begin{aligned}
& \lim _{\epsilon \downarrow 0} \epsilon^{2(r-p) /(2-p)-1} \sum_{n=1}^{\infty} n^{\frac{r}{p}-2-\frac{1}{p}} E\left\{\left|S_{n}\right|-\epsilon n^{\frac{1}{p}}\right\}_{+} \\
& =\frac{p(2-p)}{(r-p)(2 r-p-2)} E|Z|^{\frac{2(r-p)}{2-p}},
\end{aligned}
$$

where $S_{n}=X_{1}+X_{2}+\cdots+X_{n}$ and $Z$ has a normal distribution with mean 0 and variance $\sigma^{2}$.
\end{abstract}

\section{Introduction}

A finite sequence $\left\{X_{i}, 1 \leq i \leq n\right\}$ of random variables is said to be associated if for any pair of coordinatewise non-decreasing functions $f$ and $g \operatorname{Cov}\left(f\left(X_{1}, \cdots, X_{n}\right), g\left(X_{1}, \cdots, X_{n}\right)\right) \geq 0$ and a finite sequence $\left\{X_{i}, 1 \leq i \leq n\right\}$ is negatively associated(NA) if for every disjoint subsets $\mathrm{A}$ and $\mathrm{B}$ of $\{1,2, \cdots, n\}$, we have

$$
\operatorname{Cov}\left(f\left(X_{i}, i \in A\right), g\left(X_{j}, j \in B\right)\right) \leq 0,
$$

whenever $f$ on $R^{A}$ and $g$ on $R^{B}$ are coordinatewise nondecreasing functions and the covariance exists. An infinite sequence of random variables is associated(negatively associated) if every finite subfamily is associated(negatively associated). These definitions were introduced by Esary, Proschan and Walkup (1967), and Joag-Dev and Proschan(1983), respectively. As pointed out and proved by Joag-Dev and Proschan(1983)

Received July 13, 2009. Accepted August 20, 2009.

2000 Mathematics Subject Classification. 60F15, 60G50

Key words and phrases. Precise asymptotics, Complete moment convergence, Negatively associated, Berry-Esseen inequality. 
a number of well known multivariate distributions possess the NA property such as the multinomial distribution, the multivariate hypergeometric distribution, the negatively correlated normal distribution, the random sampling without replacement. Under suitable conditions, many results have been for negatively associated random variables. For example, Joag-Dev and Proschan(1983) proved the fundamental properties, Shao and $\mathrm{Su}(1999)$ showed the law of the iterated logarithm, Liang(2000) obtained complete convergence, Li and Zhang(2004) showed complete moment convergence, and $\mathrm{Fu}$ and Zhang(2007) investigated the precise rates in the law of the logarithm.

Note that in the above mentioned limit theorems, the precise asymptotics in the complete moment convergence for negatively associated sequences are little known. The aim of present work is to investigate this asymptotic behavior for NA random variables. Of course, our results has the connection with complete moment convergence. It is well known that a lot of beautiful results have been established for independent and identically distributed random variables. Heyde(1975) proved that $\lim _{\epsilon \downarrow 0} \epsilon^{2} \sum_{n=1}^{\infty} P\left(\left|S_{n}\right|>\epsilon n\right)=E X^{2}$, where $S_{n}=X_{1}+X_{2}+\cdots+X_{n}$, $E X=0$ and $E X^{2}<\infty$. For analogous results, Chen(1978) and Gut and Spataru(2000) studied the precise asymptotics in the Baum-Katz law of large numbers as $\epsilon \downarrow 0$. One of their results is as follows:

Theorem A(Gut and Spătaru(2000)) Suppose that $\left\{X_{n}, n \geq 1\right\}$ is a sequence of i.i.d. random variables with $E X_{1}=0$ and $E X_{1}^{2}=\sigma^{2}<\infty$. Then, for $1 \leq p<r$

$$
\lim _{\epsilon \downarrow 0} \epsilon^{\frac{2(r-p)}{2-p}} \sum_{n=1}^{\infty} n^{\frac{r}{p}-2} P\left\{\left|\sum_{i=1}^{n} X_{i}\right| \geq \epsilon n^{\frac{1}{p}}\right\}=\frac{p}{r-p} E|Z|^{\frac{2(r-p)}{2-p}},
$$

where $|Z|$ has a normal distribution with mean zero and variance $\sigma^{2}$.

On the other hand, Chow(1988) obtained a complete moment convergence as follows.

Theorem B(Chow(1988)) Suppose $E X_{1}=0$ and $E\left(\left|X_{1}\right|^{r}+\left|X_{1}\right| \log (1+\right.$ $\left.\left|X_{1}\right|\right)<\infty$, for $r>1,1 \leq p<2$. Then

$$
\sum_{n=1}^{\infty} n^{\frac{r}{p}-2-\frac{1}{p}} E\left(\left|S_{n}\right|-\epsilon n^{\frac{1}{p}}\right)_{+}<\infty, \epsilon>0 .
$$

$\operatorname{Li}(2006)$ also showed that the precise asymptotics result of complete moment convergence holds for i.i.d. random variables as follows. 
Theorem $\mathbf{C}(\operatorname{Li}(2006))$ Assume that $\left\{X_{i}, i \geq 1\right\}$ is a sequence of i.i.d. random variables with mean zeros and finite variances $\sigma^{2}$. Suppose $E\left|X_{1}\right|^{3}<\infty$, and for $1<p<2, r>1+\frac{p}{2}$, if $E\left|X_{1}\right|^{r}<\infty$, then we have

$$
\begin{aligned}
& \lim _{\epsilon \downarrow 0} \epsilon^{\frac{2(r-p)}{2-p}-1} \sum_{n=1}^{\infty} n^{\frac{r}{p}-2-\frac{1}{p}} E\left\{\left|\sum_{k=1}^{n} X_{k}\right|-\epsilon n^{\frac{1}{p}}\right\}_{+} \\
& =\frac{p(2-p)}{(r-p)(2 r-p-2)} E|Z|^{\frac{2(r-p)}{2-p}},
\end{aligned}
$$

where $Z$ has a normal distribution with mean zero and variance $\sigma^{2}$.

The purpose of this paper is to show that the above Theorem $\mathrm{C}$ still be true for negatively associated sequences under appropriate conditions.

\section{Preliminaries}

The next lemma is a central limit theorem for NA sequences.

Lemma 2.1(Newman(1984)) Let $\left\{X_{n}, n \geq 1\right\}$ be a strictly stationary sequence of NA random variables with $E X_{1}=0$ and $E X_{1}^{2}<\infty$. Suppose that

$$
0<\lim _{n \rightarrow \infty} \frac{E S_{n}^{2}}{n}=\sigma^{2}<\infty .
$$

holds. Then

$$
\frac{S_{n}}{\sigma \sqrt{n}} \rightarrow^{\mathcal{D}} N(0,1) \text { as } n \rightarrow \infty,
$$

where $\rightarrow^{\mathcal{D}}$ indicates convergence in distribution, $N$ a standard normal distribution and $S_{n}=\sum_{i=1}^{n} X_{i}$.

The following lemma is a type of Berry-Esseen inequality for NA sequences.

Lemma 2.2(Wang and Zhang(2006))) Let $\left\{X_{n}, n \geq 1\right\}$ be a sequence of identically distributed NA random variables with $E X_{1}=0$ and $E\left|X_{1}\right|^{3}<\infty$. Assume that

$$
\sup _{k} \sum_{j:|j-k| \geq n}\left\{-\operatorname{Cov}\left(X_{j}, X_{k}\right)\right\}=O\left(e^{-\delta n}\right) \text { for some } \delta>0
$$

and (2.1) holds.

Then, we have

$$
\Delta_{n}=\sup _{-\infty<x<\infty}\left|P\left(\frac{S_{n}}{\sigma \sqrt{n}}<x\right)-P(N<x)\right| \leq n^{-1 / 2} \log n .
$$


Remark From (2.4) it is easy to get

$$
\sup _{0<x<\infty}\left|P\left(\left|S_{n}\right| \geq x \sigma \sqrt{n}\right)-P(|N| \geq x)\right| \leq C n^{-\frac{1}{2}} \log n .
$$

Lemma 2.3(Shao(2000)) Let $\left\{X_{i}, 1 \leq i \leq n\right\}$ be a sequence of NA random variables with $E X_{i}=0$ and $E X_{i}^{2}<\infty$. Let $B_{n}=\sum_{i=1}^{n} E X_{i}^{2}$. Then, for any $x>0$ and $y>0$,

$$
\begin{aligned}
& P\left\{\max _{1 \leq k \leq n}\left|\sum_{i=1}^{k} X_{i}\right| \geq x\right\} \\
& \leq 2 P\left\{\max _{1 \leq k \leq n}\left|X_{k}\right| \geq y\right\}+4 \exp \left\{-\frac{x^{2}}{8 B_{n}}\right\}+4\left(\frac{B_{n}}{4\left(x y+B_{n}\right)}\right)^{\frac{x}{12 y}}
\end{aligned}
$$

Proposition 2.4( $\operatorname{Li(2006))~For~} 1<p<2$ and $r>1+\frac{p}{2}$, we have

$$
\begin{aligned}
& \lim _{\epsilon \downarrow 0} \epsilon^{\frac{2(r-p)}{2-p}-1} \sum_{n=1}^{\infty} n^{\frac{r}{p}-2-\frac{1}{p}+\frac{1}{2}} E\left\{|N|-\epsilon n^{\frac{1}{p}-\frac{1}{2}}\right\}_{+} \\
& =\frac{p(2-p)}{(r-p)(2 r-p-2)} E|N|^{\frac{2(r-p)}{2-p}},
\end{aligned}
$$

where $N$ is a standard normal random variable.

Proof See the proof of Proposition 3.1 in $\operatorname{Li}(2006)$.

Proposition 2.5 Let $a(\epsilon)=\epsilon^{-\frac{2 p}{2-p}}$. Assume $E\left|X_{1}\right|^{r}<\infty$. Then, for $1<p<2, r>1+\frac{p}{2}$ we have

$$
\lim _{M \rightarrow \infty} \epsilon^{\frac{2(r-p)}{2-p}-1} \sum_{n>a(\epsilon) M} n^{\frac{r}{p}-2-\frac{1}{p}} n^{\frac{1}{2}} E\left\{|N|-\epsilon n^{\frac{1}{p}-\frac{1}{2}}\right\}_{+}=0
$$

uniformly with respect to all sufficient small $0<\epsilon<1$.

Proof See the proof of Proposition 3.3 in $\operatorname{Li}(2006)$.

\section{Main Results}

Theorem 3.1 Let $\left\{X_{n}, n \geq 1\right\}$ be a sequence of identically distributed NA random variables with $E X_{1}=0$ and $E X_{1}^{2}<\infty$. Suppose $E\left|X_{1}\right|^{3}<$ $\infty$ and for $1<p<2, r>1+\frac{p}{2}, E\left|X_{1}\right|^{r}<\infty$ and let $S_{n}=\sum_{i=1}^{n} X_{i}$. If

$$
\sup _{k} \sum_{j:|j-k| \geq n}\left\{-\operatorname{Cov}\left(X_{j}, X_{k}\right)\right\}=O\left(e^{-\delta n}\right) \text { for some } \delta>0,
$$


and

$$
0<\lim _{n \rightarrow \infty} \frac{E S_{n}^{2}}{n}=\sigma^{2}<\infty
$$

then

$$
\begin{aligned}
& \lim _{\epsilon \downarrow 0} \epsilon^{\frac{2(r-p)}{2-p}-1} \sum_{n=1}^{\infty} n^{\frac{r}{p}-2-\frac{1}{p}} E\left\{\left|S_{n}\right|-\epsilon n^{\frac{1}{p}}\right\}_{+} \\
& =\frac{p(2-p)}{(r-p)(2 r-p-2)} E|Z|^{\frac{2(r-p)}{2-p}}
\end{aligned}
$$

where $Z$ has a normal distribution with mean 0 and variance $\sigma^{2}$.

Without loss of generality, we assume $\sigma^{2}=1$ in the sequel. Let $a(\epsilon):=\epsilon^{-2 p /(2-p)}$.

Proposition 3.2 Let $\left\{X_{n}: n \geq 1\right\}$ be a strictly stationary sequence of NA random variables with mean zero and variance one. Let $a(\epsilon):=$ $\epsilon^{-\frac{2 p}{2-p}}$. Assume $E\left|X_{1}\right|^{3}<\infty$ and that (3.1) and (3.2) hold. Then, for $1<p<2, r>1+\frac{p}{2}$ and all $M>2$, we have

$\lim _{\epsilon \downarrow 0} \epsilon^{\frac{2(r-p)}{2-p}-1} \sum_{n \leq a(\epsilon) M} n^{\frac{r}{p}-2-\frac{1}{p}}\left|E\left\{\left|S_{n}\right|-\epsilon n^{\frac{1}{p}}\right\}_{+}-n^{\frac{1}{2}} E\left\{|N|-\epsilon n^{\frac{1}{p}-\frac{1}{2}}\right\}_{+}\right|=0$.

Proof As in the proof of Proposition 3.2 in $\operatorname{Li}(2006)$ it is easy to see that 


$$
\begin{aligned}
& \epsilon^{\frac{2(r-p)}{2-p}-1} \sum_{n \leq a(\epsilon) M} n^{\frac{r}{p}-2-\frac{1}{p}}\left|E\left\{\left|S_{n}\right|-\epsilon n^{\frac{1}{p}}\right\}_{+}-n^{\frac{1}{2}} E\left\{|N|-\epsilon n^{\frac{1}{p}-\frac{1}{2}}\right\}_{+}\right| \\
& \leq \quad \epsilon^{\frac{2(r-p)}{2-p}-1} \sum_{n \leq a(\epsilon) M} n^{\frac{r}{p}-2-\frac{1}{p}} \mid \int_{0}^{n^{\frac{1}{p}}} P\left\{\left|S_{n}\right| \geq \epsilon n^{\frac{1}{p}}+x\right\} d x \\
& +\int_{n^{\frac{1}{p}}}^{\infty} P\left\{\left|S_{n}\right| \geq \epsilon n^{\frac{1}{p}}+x\right\} d x-n^{\frac{1}{2}}\left(\int_{0}^{n^{\frac{1}{p}-\frac{1}{2}}} P\left\{|N| \geq \epsilon n^{\frac{1}{p}-\frac{1}{2}}+x\right\} d x\right. \\
& \left.+\int_{n^{\frac{1}{p}-\frac{1}{2}}}^{\infty} P\left\{|N| \geq \epsilon n^{\frac{1}{p}-\frac{1}{2}}+x\right\} d x\right) \mid \\
& \leq \quad \epsilon^{\frac{2(r-p)}{2-p}-1} \sum_{n \leq a(\epsilon) M} n^{\frac{r}{p}-2-\frac{1}{p}+\frac{1}{2}}\left[\int_{0}^{n^{\frac{1}{p}-\frac{1}{2}}} \mid P\left\{\left|S_{n}\right| / \sqrt{n} \geq \epsilon n^{\frac{1}{p}-\frac{1}{2}}+x\right\}\right. \\
& -P\left\{|N| \geq \epsilon n^{\frac{1}{p}-\frac{1}{2}}+x\right\}\left|d x+\int_{n^{\frac{1}{p}-\frac{1}{2}}}^{\infty}\right| P\left\{\left|S_{n}\right| / \sqrt{n} \geq \epsilon n^{\frac{1}{p}-\frac{1}{2}}+x\right\} \\
& \left.-P\left\{|N| \geq \epsilon n^{\frac{1}{p}-\frac{1}{2}}+x\right\} \mid d x\right] \\
& \leq \quad \epsilon^{\frac{2(r-p)}{2-p}-1} \sum_{n \leq a(\epsilon) M} n^{\frac{r}{p}-2-\frac{1}{p}+\frac{1}{2}}\left(\Delta_{n_{1}}+\Delta_{n_{2}}\right) .
\end{aligned}
$$

From $(2.5)$ and $1<p<2$

$$
\begin{aligned}
\Delta_{n_{1}} & =\int_{0}^{n^{\frac{1}{p}-\frac{1}{2}}}\left|P\left\{\left|S_{n}\right| / \sqrt{n} \geq \epsilon n^{\frac{1}{p}-\frac{1}{2}}+x\right\}-P\left\{|N| \geq \epsilon n^{\frac{1}{p}-\frac{1}{2}}+x\right\}\right| d x \\
& \leq n^{\frac{1}{p}-\frac{1}{2}} \sup _{0<x<\infty}\left|P\left\{\left|S_{n}\right| / \sqrt{n} \geq x\right\}-P\{|N| \geq x\}\right| \\
& \leq C n^{\frac{1}{p}-\frac{1}{2}}\left(n^{-\frac{1}{2}} \log n\right) \rightarrow 0 \text { as } n \rightarrow \infty
\end{aligned}
$$

From central limit theorem of NA random variables(see Lemma 2.1), for $1<\alpha<2, E\left|S_{n} / \sqrt{n}\right|^{\alpha}<\infty$. This entails, by virtues of Markov's inequality, that for $x \geq n^{\frac{1}{p}-\frac{1}{2}}$,

$$
\mid P\left\{\left|S_{n}\right| / \sqrt{n} \geq \epsilon n^{\frac{1}{p}-\frac{1}{2}}+x\right\}-P\left\{|N| \geq \epsilon n^{\frac{1}{p}-\frac{1}{2}}+x\right\} \leq C x^{-\alpha} .
$$


So we get

$$
\begin{aligned}
\Delta_{n_{2}} & =\int_{n^{\frac{1}{p}-\frac{1}{2}}}^{\infty} \mid P\left\{\left|S_{n}\right| / \sqrt{n} \geq \epsilon n^{\frac{1}{p}-\frac{1}{2}}+x\right\}-P\left\{|N| \geq \epsilon n^{\frac{1}{p}-\frac{1}{2}}+x\right\} d x \\
& \leq \int_{n^{\frac{1}{p}-\frac{1}{2}}}^{\infty} C x^{-\alpha} d x \\
& \leq C n^{\left(\frac{1}{p}-\frac{1}{2}\right)(1-\alpha)} \rightarrow 0 \text { as } n \rightarrow \infty \text { for } 1<\alpha<2,1<p<2 .
\end{aligned}
$$

Denote $\Delta_{n}=\Delta_{n_{1}}+\Delta_{n_{2}}$. By Toeplitz's lemma it follows that

$$
m^{-\frac{r}{p}+\frac{1}{p}+\frac{3}{2}} \sum_{n=1}^{m} n^{\frac{r}{p}-2-\frac{1}{p}+\frac{1}{2}} \Delta_{n} \rightarrow 0 \text { as } m \rightarrow \infty .
$$

We have

$$
\begin{aligned}
\lim _{\epsilon \downarrow 0} \epsilon^{\frac{2(r-p)}{2-p}-1} & \sum_{n \leq a(\epsilon) M} n^{\frac{r}{p}-2-\frac{1}{p}+\frac{1}{2}}\left|E\left\{\left|S_{n}\right|-\epsilon n^{\frac{1}{p}}\right\}_{+}-n^{\frac{1}{2}} E\left\{|N|-\epsilon n^{\frac{1}{p}-\frac{1}{2}}\right\}_{+}\right| \\
=\quad & \lim _{\epsilon \downarrow 0} \epsilon^{\frac{2(r-p)}{2-p}-1} \sum_{n \leq a(\epsilon) M} n^{\frac{r}{p}-2-\frac{1}{p}+\frac{1}{2}} \Delta_{n} \\
\leq \quad & \lim _{\epsilon \downarrow 0} \epsilon^{\frac{2(r-p)}{2-p}-1}[a(\epsilon) M]^{\frac{r}{p}-\frac{1}{2}-\frac{1}{p}}[a(\epsilon) M]^{-\frac{r}{p}+\frac{1}{2}+\frac{1}{p}} \\
& \times \sum_{n \leq a(\epsilon) M} n^{\frac{r}{p}-2-\frac{1}{p}+\frac{1}{2}} \Delta_{n}=0 .
\end{aligned}
$$

Proposition 3.3 Let $\left\{X_{n}, n \geq 1\right\}$ be a sequence of identically distributed NA random variables with mean zero and finite variance. Assume $E\left|X_{1}\right|^{r}<\infty$ for $1<p<2, r>1+\frac{p}{2}$. Then we have

$$
\lim _{M \rightarrow \infty} \lim _{\epsilon \downarrow 0} \sup \epsilon^{\frac{2(r-p)}{2-p}-1} \sum_{n>a(\epsilon) M} n^{\frac{r}{p}-2-\frac{1}{p}} E\left\{\left|S_{n}\right|-\epsilon n^{\frac{1}{p}}\right\}_{+}=0 .
$$


Proof For $1<p<2$ and $1+\frac{p}{2}<r<2$, noting that $E X_{1}^{2}<\infty$, we have

$$
\begin{aligned}
I & =: \sum_{n>a(\epsilon) M} n^{\frac{r}{p}-2-\frac{1}{p}} E\left\{\left|S_{n}\right|-\epsilon n^{\frac{1}{p}}\right\}_{+} \\
& =\sum_{n>a(\epsilon) M} n^{\frac{r}{p}-2-\frac{1}{p}} \int_{\epsilon n^{\frac{1}{p}}}^{\infty} P\left\{\left|S_{n}\right| \geq x\right\} d x \\
& \leq \sum_{n>a(\epsilon) M} n^{\frac{r}{p}-2-\frac{1}{p}} \int_{\epsilon n^{\frac{1}{p}}}^{\infty} \frac{n E X_{1}^{2}}{x^{2}} \\
& \leq C \sum_{n>a(\epsilon) M} n^{\frac{r}{p}-1-\frac{1}{p}}\left(\epsilon n^{\frac{1}{p}}\right)^{-1} \\
& =C \sum_{n>a(\epsilon) M} \epsilon^{-1} n^{\frac{r}{p}-1-\frac{2}{p}} \leq C \epsilon^{-1}(a(\epsilon) M)^{\frac{r}{p}-\frac{2}{p}} \\
& =C \epsilon^{-\frac{2(r-p)}{2-p}+1} M^{\frac{r-2}{p}} .
\end{aligned}
$$

So it follows that, for $1<p<2$ and $1+\frac{p}{2}<r<2$, we get

$$
\lim _{M \rightarrow \infty} \lim _{\epsilon \downarrow 0} \sup \epsilon^{\frac{2(r-p)}{2-p}-1} I \leq \lim _{M \rightarrow \infty} C M^{\frac{r-2}{p}}=0 .
$$

Let $\lambda=\left(E X_{1}^{2}\right)^{-\frac{1}{2}}$ and $B_{n}=\sum_{i=1}^{n} E X_{i}^{2}=n \lambda^{-2}$.

For $1<p<2$ and $r \geq 2$, using Lemma 2.3 with $y=\beta x$ (whose value will be specified later), we have

$$
\begin{aligned}
I \leq & \sum_{n>a(\epsilon) M} n^{\frac{r}{p}-2-\frac{1}{p}} \int_{\epsilon n^{\frac{1}{p}}}^{\infty}\left[2 P\left\{\max _{1 \leq i \leq n}\left|X_{i}\right|>\beta x\right\}\right. \\
& \left.+4 \exp \left\{-\frac{x^{2} n^{-1} \lambda^{2}}{8}\right\}+4\left\{\frac{1}{4\left(\beta x^{2} n^{-1} \lambda^{2}+1\right)}\right\}^{\frac{1}{12 \beta}}\right] d x \\
= & \sum_{n>a(\epsilon) M} n^{\frac{r}{p}-2-\frac{1}{p}} \int_{\epsilon n^{\frac{1}{p}}}^{\infty}\left(I_{1}+I_{2}+I_{3}\right) d x .
\end{aligned}
$$


For $I_{1}$, we have

$$
\begin{aligned}
& \sum_{n>a(\epsilon) M} n^{\frac{r}{p}-2-\frac{1}{p}} \int_{\epsilon n^{\frac{1}{p}}}^{\infty} I_{1} d x \\
\leq & 2 \sum_{n>a(\epsilon) M} n^{\frac{r}{p}-2-\frac{1}{p}} \int_{\epsilon n^{\frac{1}{p}}}^{\infty} n P\left\{\left|X_{1}\right| \geq \beta x\right\} d x \\
\leq & 2 \sum_{n>a(\epsilon) M} n^{\frac{r}{p}-1-\frac{1}{p}} \int_{\beta \epsilon n^{\frac{1}{p}}}^{\infty} P\left\{\left|X_{1}\right| \geq x\right\} d x \\
\leq & C \int_{a(\epsilon) M}^{\infty} t^{\frac{r}{p}-1-\frac{1}{p}} \int_{\beta \epsilon t^{\frac{1}{p}}}^{\infty} P\left\{\left|X_{1}\right| \geq x\right\} d x d t \\
\leq & C \int_{a(\epsilon) M-1}^{\infty} t^{\frac{r}{p}-1-\frac{1}{p}} \int_{\beta \epsilon t^{\frac{1}{p}}}^{\infty} P\left\{\left|X_{1}\right| \geq x\right\} d x d t \\
\leq & C \int_{\frac{a(\epsilon) M}{2}}^{t^{\frac{r}{p}-1-\frac{1}{p}}} \int_{\beta \epsilon t^{\frac{1}{p}}}^{\infty} P\left\{\left|X_{1}\right| \geq x\right\} d x d t \\
\leq & C \epsilon^{-r+1} \int_{\beta \epsilon\left[\frac{a(\epsilon) M}{2}\right]^{\frac{1}{p}}}^{\infty} y^{r-2} \int_{y}^{\infty} P\left\{\left|X_{1}\right| \geq x\right\} d x d y \\
\leq & C \epsilon^{-r+1} \int_{\beta \epsilon\left[\frac{a(\epsilon) M}{2}\right]^{\frac{1}{p}}}^{\infty}\left(\int_{\beta \epsilon\left[\frac{a(\epsilon) M}{2}\right]^{\frac{1}{p}}}^{\infty} y^{r-2} d y\right) P\left\{\left|X_{1}\right| \geq x\right\} d x
\end{aligned}
$$

letting $A=\beta \epsilon\left[\frac{a(\epsilon) M}{2}\right]^{\frac{1}{p}}$

$$
\begin{aligned}
& \leq C \epsilon^{-r+1}(r-1)^{-1} \int_{A}^{\infty} x^{r-1} P\left\{\left|X_{1}\right| \geq x\right\} d x \\
& \leq \frac{C}{r-1} \epsilon^{-r+1} \int_{A}^{\infty} x^{r-1} \sum_{k=[A]} P\left(k \leq\left|X_{1}\right|<k+1\right) d x \\
& \leq \frac{C}{r-1} \epsilon^{-r+1} \sum_{k=[A]}^{\infty} P\left(k \leq\left|X_{1}\right|<k+1\right) \int_{[A]}^{k+1} x^{r-1} d x \\
& \leq C \frac{\epsilon^{-r+1}}{r-1} \sum_{k=[A]}^{\infty}(k+1)^{r} P\left(k \leq\left|X_{1}\right|<k+1\right) \\
& \leq C \frac{\epsilon^{-r+1}}{r-1} E\left\{\left|X_{1}\right|^{r} I\left(\left|X_{1}\right| \geq A\right)\right\} .
\end{aligned}
$$


Note $E\left|X_{1}\right|^{r} I\left(\left|X_{1}\right| \geq A\right) \rightarrow 0$ as $M \rightarrow \infty$. Hence, for $r=2$

$$
\begin{aligned}
\lim _{M \rightarrow \infty} \lim _{\epsilon \downarrow 0} \epsilon^{\frac{2(r-p)}{2-p}-1} & \sum_{n>a(\epsilon) M} n^{\frac{r}{p}-2-\frac{1}{p}} \int_{\epsilon n^{\frac{1}{p}}}^{\infty} I_{1} d x \\
\leq & C \lim _{M \rightarrow \infty} \lim _{\epsilon \downarrow 0} E\left\{\left|X_{1}\right|^{2} I\left(\left|X_{1}\right| \geq A\right)\right\}=0 .
\end{aligned}
$$

By $E\left|X_{1}\right|^{r}<\infty$ it follows that

$$
\begin{aligned}
& \lim _{M \rightarrow \infty} \lim _{\epsilon \downarrow 0} \epsilon^{\frac{2(r-p)}{2-p}-1} \sum_{n>a(\epsilon) M} n^{\frac{r}{p}-2-\frac{1}{p}} \int_{\epsilon n^{\frac{1}{p}}}^{\infty} I_{1} d x \\
& \leq \quad C \lim _{\epsilon \downarrow 0} \epsilon^{\frac{p(r-2)}{2-p}}=0 \text { for } r>2 .
\end{aligned}
$$

Now we estimate $I_{2}$, for $r>1+\frac{p}{2}$, we have

$$
\begin{aligned}
\sum_{n>a(\epsilon) M} n^{\frac{r}{p}-2-\frac{1}{p}} & \int_{\epsilon n^{\frac{1}{p}}}^{\infty} I_{2} d x \\
& =\quad C \sum_{n>a(\epsilon) M} n^{\frac{r}{p}-2-\frac{1}{p}} \int_{\epsilon n^{\frac{1}{p}}}^{\infty} \exp \left(-C x^{2} n^{-1}\right) d x \\
& \leq \quad C \int_{a(\epsilon) M / 2}^{\infty} y^{\frac{r}{p}-2-\frac{1}{p}} \int_{\epsilon y^{\frac{1}{p}}}^{\infty} \exp \left(-C x^{2} y^{-1}\right) d x d y \\
& \quad C \int_{\frac{a(\epsilon) M}{2}}^{\infty} y^{\frac{r}{p}-2-\frac{1}{p}+\frac{1}{2}} \int_{\epsilon^{2} y^{\frac{2}{p}-1}}^{\infty} t^{-\frac{1}{2}} \exp (-C t) d t d y \\
\leq & C \int_{\left(\frac{M}{2}\right)^{\frac{2-p}{p}}}^{\infty}\left(\int_{\frac{a(\epsilon) M}{2}}^{\left(\frac{t}{\epsilon^{2}}\right)^{\frac{p}{2-p}}} y^{\frac{r}{p}-2-\frac{1}{p}+\frac{1}{2}} d y\right) t^{-\frac{1}{2}} \exp (-C t) d t \\
\leq & C\left(\frac{r}{p}-\frac{1}{2}-\frac{1}{p}\right)^{-1} \epsilon^{-\frac{2(r-p)}{2-p}+1} \int_{\left(\frac{M}{2}\right)^{\frac{2-p}{p}}}^{\infty} t^{\frac{r-2}{2-p}} \exp (-C t) d t .
\end{aligned}
$$

It follows that, for $1<p<2$ and $r>1+\frac{p}{2}$

$$
\begin{aligned}
\lim _{M \rightarrow \infty} \lim _{\epsilon \downarrow 0} \sup \epsilon^{\frac{2(r-p)}{2-p}-1} \sum_{n>a(\epsilon) M} n^{\frac{r}{p}-2-\frac{1}{p}} \int_{\epsilon n^{\frac{1}{p}}}^{\infty} I_{2} d x \\
\leq C \lim _{M \rightarrow \infty} \int_{\left(\frac{M}{2}\right)^{\frac{2-p}{p}}}^{\infty} t^{\frac{r-2}{2-p}} \exp (-C t) d t=0 .
\end{aligned}
$$

For $I_{3}$ under the assumption $r>1+\frac{p}{2}$, which means that $\frac{(2-p)}{12(r-p)}<\frac{1}{6}$.

So we choose $\beta$ such that $0<\beta<\frac{(2-p)}{12(r-p)}$. 
Then

$$
\begin{aligned}
& \sum_{n>a(\epsilon) M} n^{\frac{r}{p}-2-\frac{1}{p}} \int_{\epsilon n^{\frac{1}{p}}}^{\infty} I_{3} d x \\
\leq & 4 \int_{\frac{a(\epsilon) M}{2}}^{\infty} y^{\frac{r}{p}-2-\frac{1}{p}} \int_{\epsilon y^{\frac{1}{p}}}^{\infty}\left(\frac{1}{4\left(\beta x^{2} y^{-1} \lambda^{2}+1\right)}\right)^{\frac{1}{12 \beta}} d x d y \\
\leq & C \int_{\frac{a(\epsilon) M}{2}}^{\infty} y^{\frac{r}{p}-2-\frac{1}{p}} \int_{\epsilon y^{\frac{1}{p}}}^{\infty}\left(\frac{y}{4\left(\beta x^{2} \lambda^{2}\right)}\right)^{\frac{1}{12 \beta}} d x d y \\
\leq & C \int_{\frac{a(\epsilon) M}{2}}^{\infty} y^{\frac{r}{p}-2-\frac{1}{p}+\frac{1}{12 \beta}} \int_{\epsilon y^{\frac{1}{p}}}^{\infty} x^{-\frac{1}{6 \beta}} d x d y \\
\leq & C \int_{\frac{a(\epsilon) M}{2}}^{\infty} y^{\frac{r}{p}-2-\frac{1}{p}+\frac{1}{12 \beta}-\frac{1}{6 \beta p}} d y \\
\leq & C \int_{\frac{a(\epsilon) M}{2}}^{\infty} y^{\frac{r-2 p}{p}-\frac{2-p}{12 \beta p}} d y .
\end{aligned}
$$

Hence, for $1<p<2$ and $r>1+\frac{p}{2}$

$$
\begin{aligned}
& \lim _{M \rightarrow \infty} \lim _{\epsilon \downarrow 0} \sup -\left(\frac{r-2 p}{p}-\frac{2-p}{12 \beta p}+1\right)^{-1}\left(\epsilon^{\frac{-2 p}{2-p}}\right)^{\frac{r-2 p}{p}-\frac{2-p}{12 \beta p}+1} \\
& \times\left(\frac{M}{2}\right)^{\frac{r-2}{p}-\frac{2-p}{12 \beta p}+1}=0
\end{aligned}
$$

since $\frac{r-2 p}{p}-\frac{2-p}{12 \beta p}+1<0$.

Combining Propositions 2.5 and 3.3 we have:

Proposition 3.4 Let $\left\{X_{n}: n \geq 1\right\}$ be a sequence of identically distributed NA random variables with mean zero and variance one. Assume $E\left|X_{1}\right|^{r}<\infty$. Then, for $1<p<2, r>1+\frac{p}{2}$, we have

$\lim _{M \rightarrow \infty} \lim _{\epsilon \downarrow 0} \sup \epsilon^{\frac{2(r-p)}{2-p}-1} \sum_{n>a(\epsilon) M} n^{\frac{r}{p}-2-\frac{1}{p}}\left|E\left\{\left|S_{n}\right|-\epsilon n^{\frac{1}{p}}\right\}_{+}-n^{\frac{1}{2}} E\left\{|N|-\epsilon n^{\frac{1}{p}-\frac{1}{2}}\right\}_{+}\right|=0$

where $N$ is a standard normal random variable.

Proof of Theorem 3.1 Theorem 3.1 is proved via Proposition 2.4, 3.2 and 3.4 .

\section{ACKNOWLEDGEMENTS.}

This work was supported by Howon University Research Fund in 2009. 


\section{References}

[1] Esary, J., Proschan, F. and Walkup(1967) Association of random variables with application, Ann. Math. Stat. 38 1466-1474

[2] Chen, R.(1978) A remark on the tail probability of distribution, J. Multivariate Anal. 8 328-333

[3] Chow, Y.S.(1988) On the rate of moment convergence of sample sums and extremes, Bull. Inst. Math. Acad. Sinica 16 177-201

[4] Fu, K.F. and Zhang, L.X.(2007) Precise rates in the law of the logarithm for negatively associated random variables, Comput. Math. Appl. 54 687-698

[5] Gut, A. and Spataru, A.(2000) Precise asymptotics in the Baum-Katz and Davis law of large numbers, J. Math. Anal. Appl. 248 233-246

[6] Heyde, C.C(1975) A supplement to the strong law of large numbers, J. Appl. Probab. 12 173-175

[7] Joag-Dev, K. and Proschan, F.(1983) Negative association of random variables with applications, Ann. Statist. 11 286-295

[8] Li, Yun-Xia (2006) Precise asymptotics in complete moment convergence of moving average processes, Statist. Probab. Lett. 76 1305-1315

[9] Li, Y.X. and Zhang, L.X.(2004) Complete moment convergence of movingaverage processes under dependence assumptions, Statist. Probab. Lett. 70 191197

[10] Liang, H.Y.(2000) Complete convergence for weighted sums of negatively associated random variables, Statist. Probab. Lett. 48 317-325

[11] Liu, W.D. and Lin, Z.Y.(2006) Precise asymptotics for a new kind of complete moment convergence, Statist. Probab. Lett. 76 1787-1799

[12] Newman, C.M.(1984) Asymptotic independence and limit theorems for positively and negatively dependent random variables, in: Y.L.Tong, ed, IMS Vol. 5 pp. $127-140$

[13] Shao, Q.M. and Su, C.(1999) The law of the iterated logarithm for negatively associated random variables, Stochastic Process Appl. 83 139-148

[14] Shao, Q.M.(2000) A comparison on maximum inequalites between negatively associated and independent random variables, J. Theort. Probab. 13 343-356

[15] Wang, J.F. and Zhang, L.X. (2006) A Berry-Esseen theorem for weakly negatively dependent random variables and its applications, Acta Math. Hungar. 110 293308

Department of Computer Science, Howon University

Chonbuk 573-718, Korea

E-mail : khhan@howon.ac.kr 